\title{
EDITORIAL
}

\section{Defining Functional Decline, Characteristics \\ of Admitted Older Adults and Comparing Alternate Versions of the MoCA}

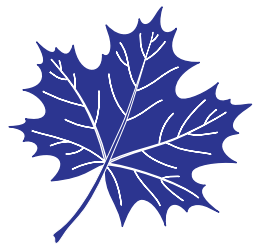

DOI:http://dx.doi.org/10.5770/cgj.19.232

Our current issue has several original research articles examining issues ranging from functional decline in trauma patients, ${ }^{(1)}$ admitted versus non-admitted older adults referred to internal medicine, ${ }^{(2)}$ and the difficulty of different versions of the MoCA. ${ }^{(3)}$

Our first article, by J. Perry, ${ }^{(1)}$ presents the results of a national survey of geriatricians designed for two purposes: to define functional decline and to provide insight into the sensitivity required for a useful clinical decision tool. This survey was rigorously developed and will provide guidance for the future study of the effects of trauma on the functional abilities of vulnerable seniors.

Dr. K. Rockwood ${ }^{(2)}$ conducted a pilot study of older adults referred to internal medicine in the emergency department setting. This study was able to show differences in the various components of frailty in patients who were admitted to hospital as opposed to being discharged home. This pilot work will help guide the future study of the intersection between acute medical illness and frailty in the older adult population.

Our final original research article compares the difficulty of alternate versus original items in the Montreal Cognitive Assessment (MoCA). The study utilized a Rasch analysis and was able to demonstrated significant heretofore unrecognized differences. ${ }^{(3)}$
In addition, we also have provided the abstracts ${ }^{(4)}$ presented at the September 2015 Canadian Association of Geriatric Psychiatry meeting in Vancouver, British Columbia, Canada.

Enjoy!

Dr. Kenneth M. Madden Editor-in-Chief Canadian Geriatrics Journal

\section{REFERENCES}

1. Abdulaziz K, Perry JJ, Taljaard M, et al. National survey of geriatricians to define functional decline in elderly people with minor trauma. CGJ. 2016;19(1):2-8.

2. Hominick K, McLeod V, Rockwood K. Characteristics of older adults admitted to hospital versus those discharged home, in emergency department patients referred to internal medicine. CGJ. 2016;19(1):9-14.

3. Lebedeva E, Huang M, Koski L. Comparison of alternate and original items on the montreal cognitive assessment. CGJ. 2016;19(1):15-18.

4. Canadian Academy of Geriatric Psychiatry 2015 Annual Scientific Meeting Abstracts. CGJ. 2016;19(1):19-32. 